(C) Published 2014. This article is a U.S. Government work and is in the public domain in the USA DOI: $10.1111 /$ sltb.12130

\title{
State of Innovation in Suicide Intervention Research with Military Populations
}

\author{
Kenneth R. Conner, PsyD, MPH, and Kelsey Simons, PhD, LMSW
}

\begin{abstract}
A systematic search was performed to identify active, externally funded randomized controlled trials (RCTs) that target suicidal ideation or behavior as a primary or secondary outcome among U.S. military service members, guardreservists, and veterans. Twenty-three studies were identified, most funded by the U.S. Department of Defense or U.S. Department of Veterans Affairs. Several innovations were identified based on departures from or modernizations of usual practices and included the targeting of suicide deaths or attempts as primary outcome, delivery of interventions through technology and/or outside clinical settings, and examinations of rarely studied treatments.
\end{abstract}

Since the wars in Afghanistan and Iraq began, also known as Operation Enduring Freedom $(\mathrm{OEF})$ and Operation Iraqi Freedom (OIF), few health topics have garnered more attention and concern from the U.S. public, the media, or elected officials than suicide among military service members and veterans. The U.S. Veterans Administration (VA) and U.S. Department of Defense (DoD), among other agencies and groups,

Kenneth R. Conner and Kelsey Simons, VISN 2 Center of Excellence for Suicide Prevention, Canandaigua VA Medical Center, Canandaigua, NY, USA, and Department of Psychiatry, University of Rochester Medical Center, Rochester, NY, USA.

This work was supported by the Canandaigua VA Medical Center, Center of Excellence for Suicide Prevention. The contents do not represent the views of the U.S. Department of Veterans Affairs or the United States Government.

An earlier version of this study was presented at the Department of Defense/Veterans Administration Suicide Prevention Summit: Innovative Practices in Research. Washington, DC, December 13, 2013.

Address correspondence to Kelsey Simons, Center of Excellence for Suicide Prevention, Canandaigua VAMC, 400 Fort Hill Ave., Canandaigua, NY 14424; E-mail: Kelsey.Simons@ va.gov have responded with numerous programs and initiatives including considerable investment in intervention studies that seek to prevent or reduce suicide-related outcomes. This research is critical because current guidelines for assessment and management of suicide risk in U.S. military populations are based on limited evidence (The Assessment \& Management of Risk for Suicide Working Group, 2013).

Innovation is one of the select few criteria for judging the quality of proposed intervention studies; yet compared with other characteristics of research (e.g., soundness of methodology), it seems difficult to operationalize. Merriam-Webster (Innovation, 2014) defines innovation as the "act or process of introducing new ideas, devices, or methods." The key word in this definition is "new," although this is a high bar to get over. Synonyms for innovation that may more readily be applied to active intervention research including "departure" and "modernization." Departures or modernizations in intervention research include, but are not limited to, what interventionists are trying to change (i.e., the outcome studied), how they are trying to change it (i.e., the intervention studied), and with what (or where) they are trying to bring about 
change (i.e., modality or setting that intervention is delivered).

There were two purposes of the current review. The first was to systematically identify active intervention research studies addressing suicidal ideation and/or behavior in military populations, including current military service members, reservists, and veterans. The second was to identify examples of innovations (i.e., departures or modernizations) in the outcomes studied, the interventions tested, and the modality or setting in which interventions are delivered, with the idea that innovations are most likely to be observed in recent (i.e., active) research.

\section{METHODS}

Active studies were eligible, including investigations that had been launched and studies that were poised to start pending local approvals (e.g., internal review board). Studies were also included when they: (1) were externally funded; (2) included random assignment to experimental condition (s) and one or more comparison conditions; (3) included a suicide-related measure as a primary or secondary outcome including standard outcomes of suicidal ideation, suicide attempt, or suicide and/or closely related nonstandard outcomes such as hospitalization for suicide risk; and (4) involved participants that were active U.S. military service members, guard-reservists, or veterans. If a study included civilians, it was eligible if it appeared to be a majority military sample. Demonstration projects and other nonresearch activities were excluded as were studies that: (1) did not use a randomized controlled design; (2) had completed all participant recruitment and follow-up assessments (i.e., study no longer active); (3) were internally funded, typically small pilots; (4) included no suicide-related measure as a primary or secondary outcome, typically studies of suicide risk factors (e.g., depression); and (5) involved industry sponsored pharmaceutical trials.
Studies of nonmilitary samples were also not eligible.

The search was conducted in November 2013 using the following primary databases and resources: (1) the National Institutes of Health's ClinicalTrials.gov (http://clinicaltrials.gov/) and Research Portfolio Online Reporting Tools (RePORTER; http://projectreporter.nih.gov/reporter.cfm); (2) 2012 and 2013 conference abstracts of the American Association of Suicidology (i.e., AAS program books) and the American Psychological Association (i.e., PsycEXTRA database); and (3) 2012-2013 research articles indexed in MEDLINE, PsychINFO, PsycARTICLES, or SocINDEX. Search terms for databases included "Veterans or military" AND "treatment, intervention, RCT, or randomized" AND "suicide." Supplemental searches were also performed on the DoDsponsored Military Suicide Research Consortium (MSRC) Web site; VA Veterans Integrated Service Network Mental Illness Research, Education \& Clinical Center (VISN 19 MIRECC) Web site; presentations given during the VA Health Services Research and Development service (HSR\&D) suicide prevention research meeting held on September 9, 2013, in Washington, DC; and through e-mail inquiries to suicide intervention researchers examining military populations.

The search identified 23 studies that met the aforementioned inclusion-exclusion criteria. Descriptive information on these studies is provided in Table 1 , including the principal investigator (PI), PI affiliation, study sponsor, and title. The most common full or partial sponsors are $\operatorname{DoD}(N=13)$ and VA $(N=9)$.

\section{RESULTS}

Table 2 contains information regarding the identified studies' methodology, including the targeted sample size, a brief description of the sample, all primary outcomes, up to two secondary outcomes, interventions used, and the intervention set- 


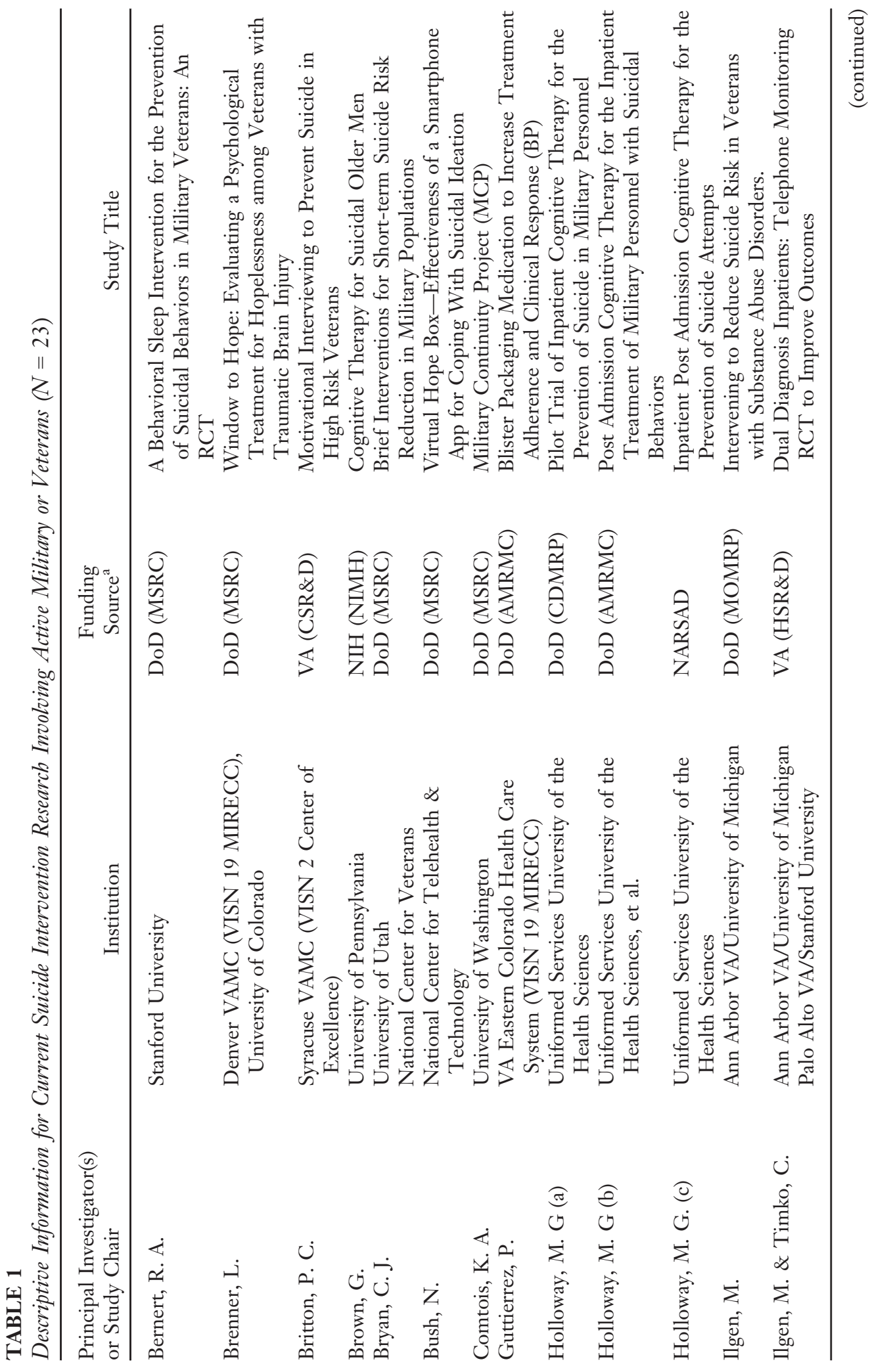




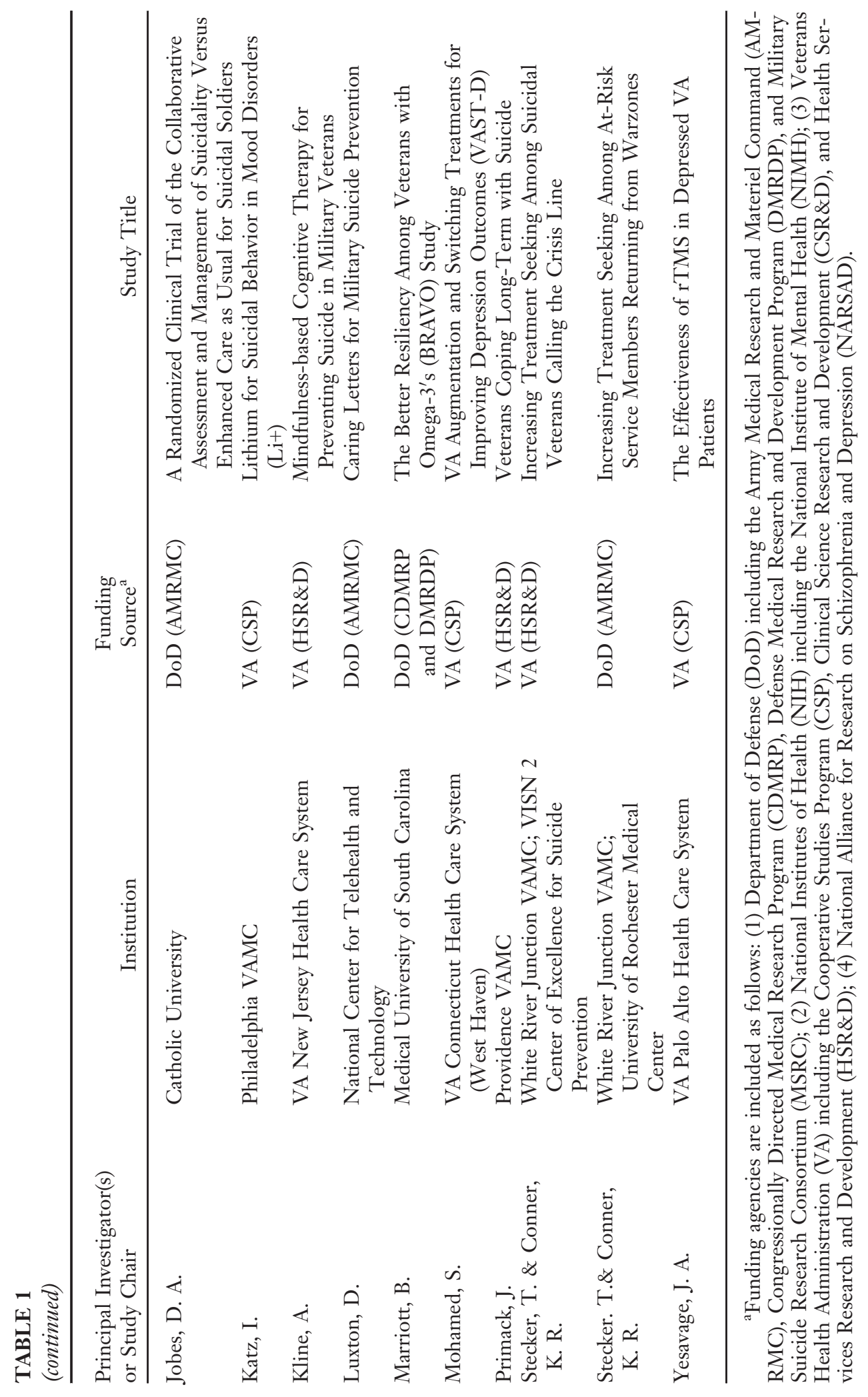




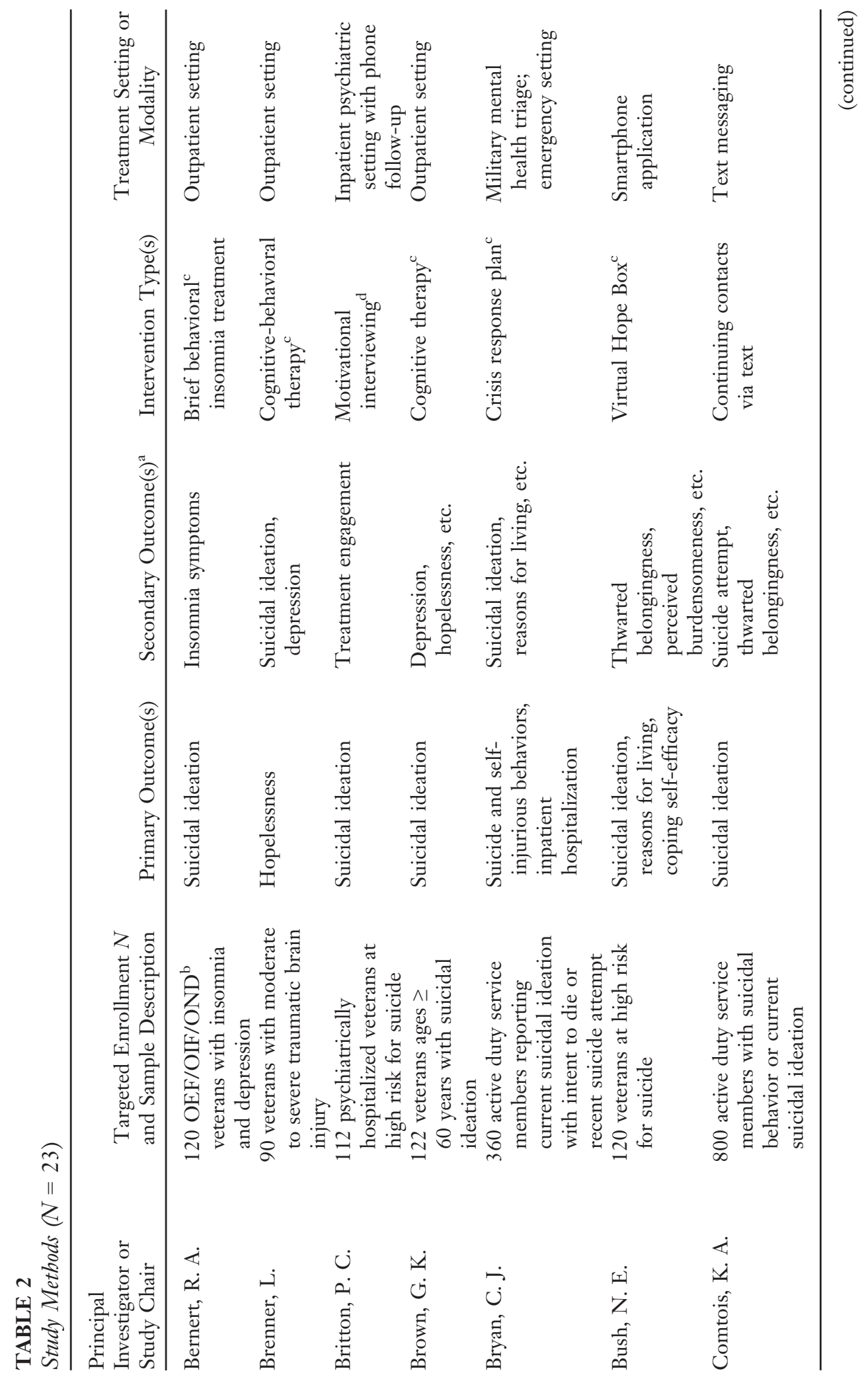




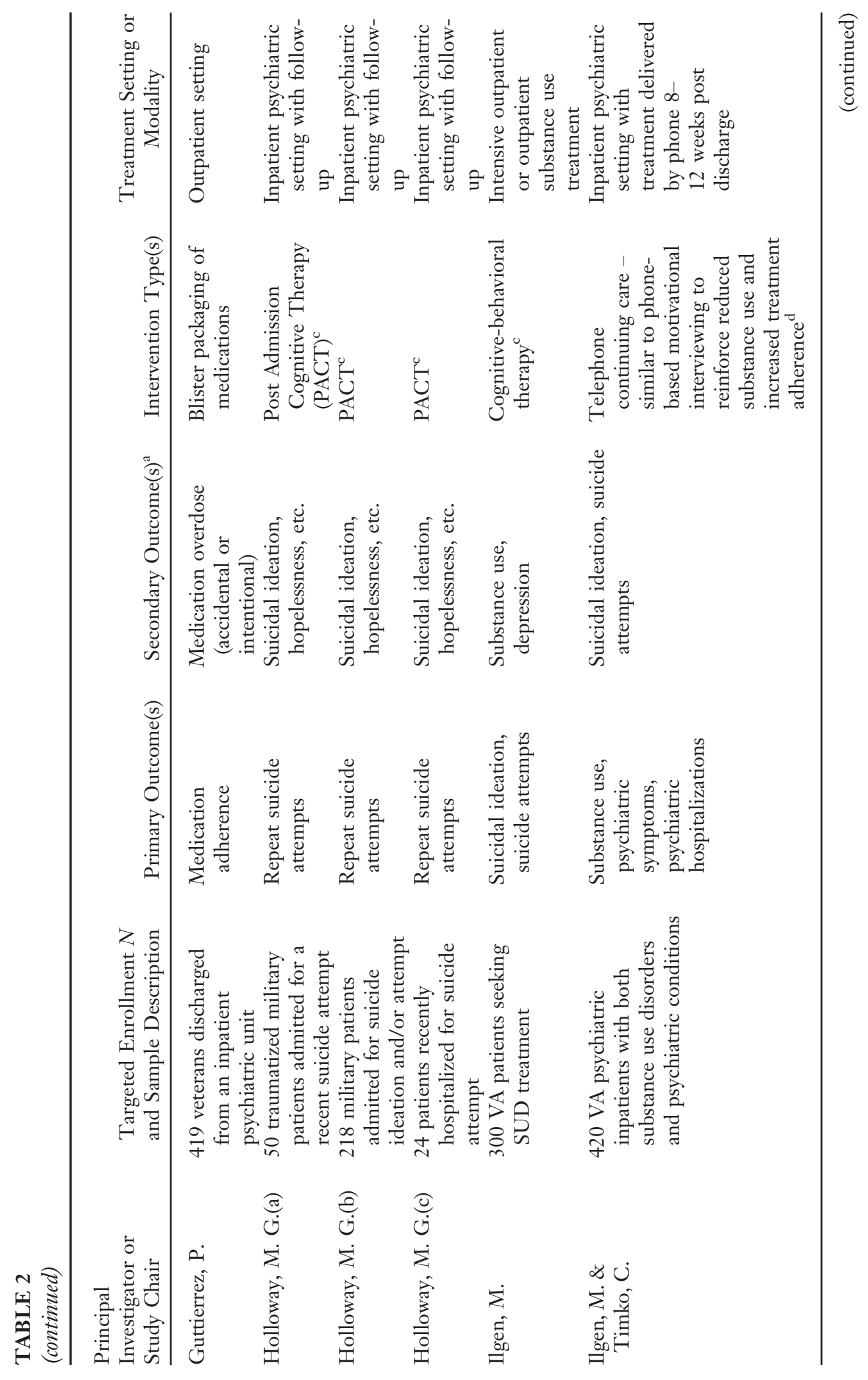




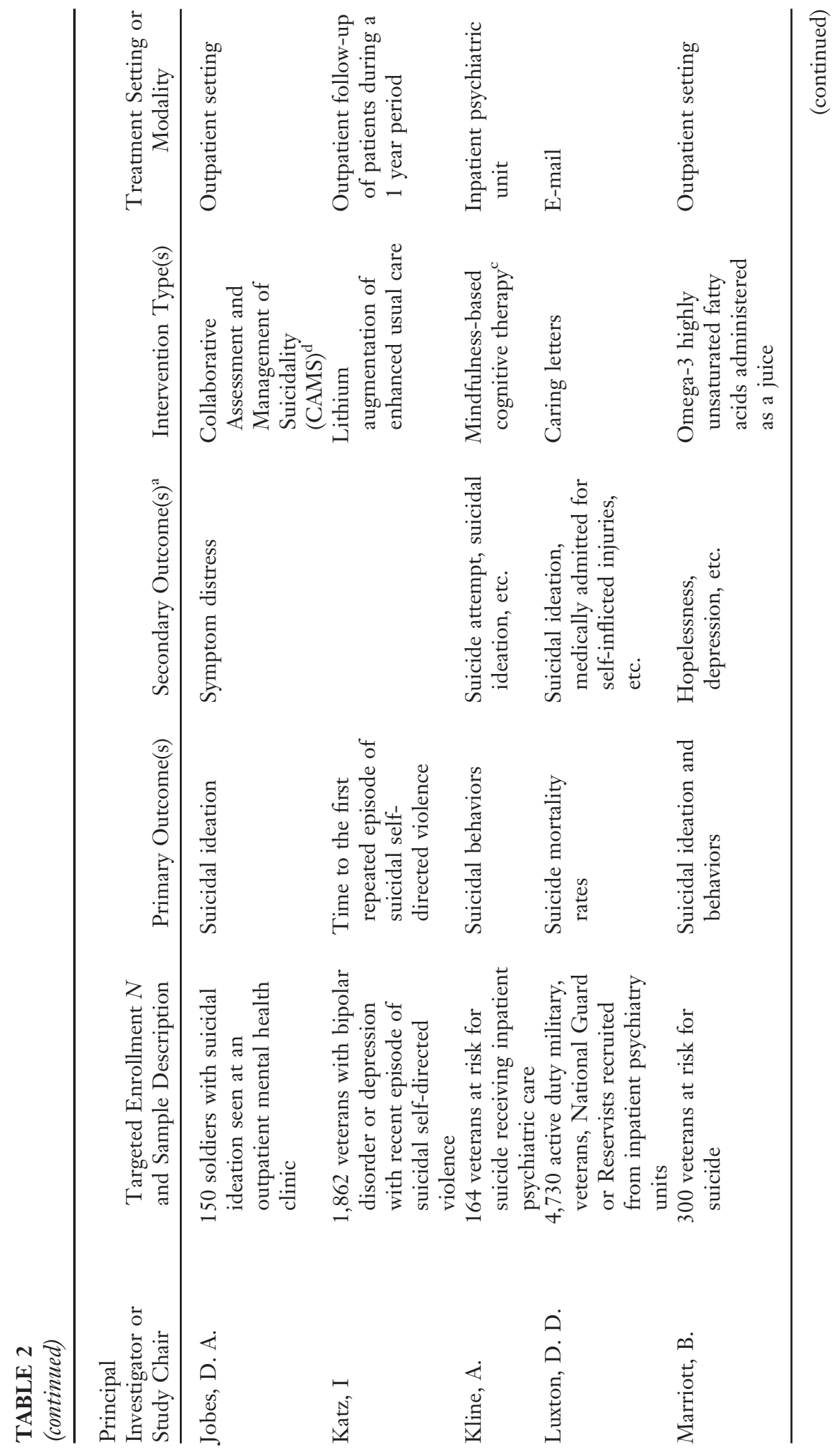




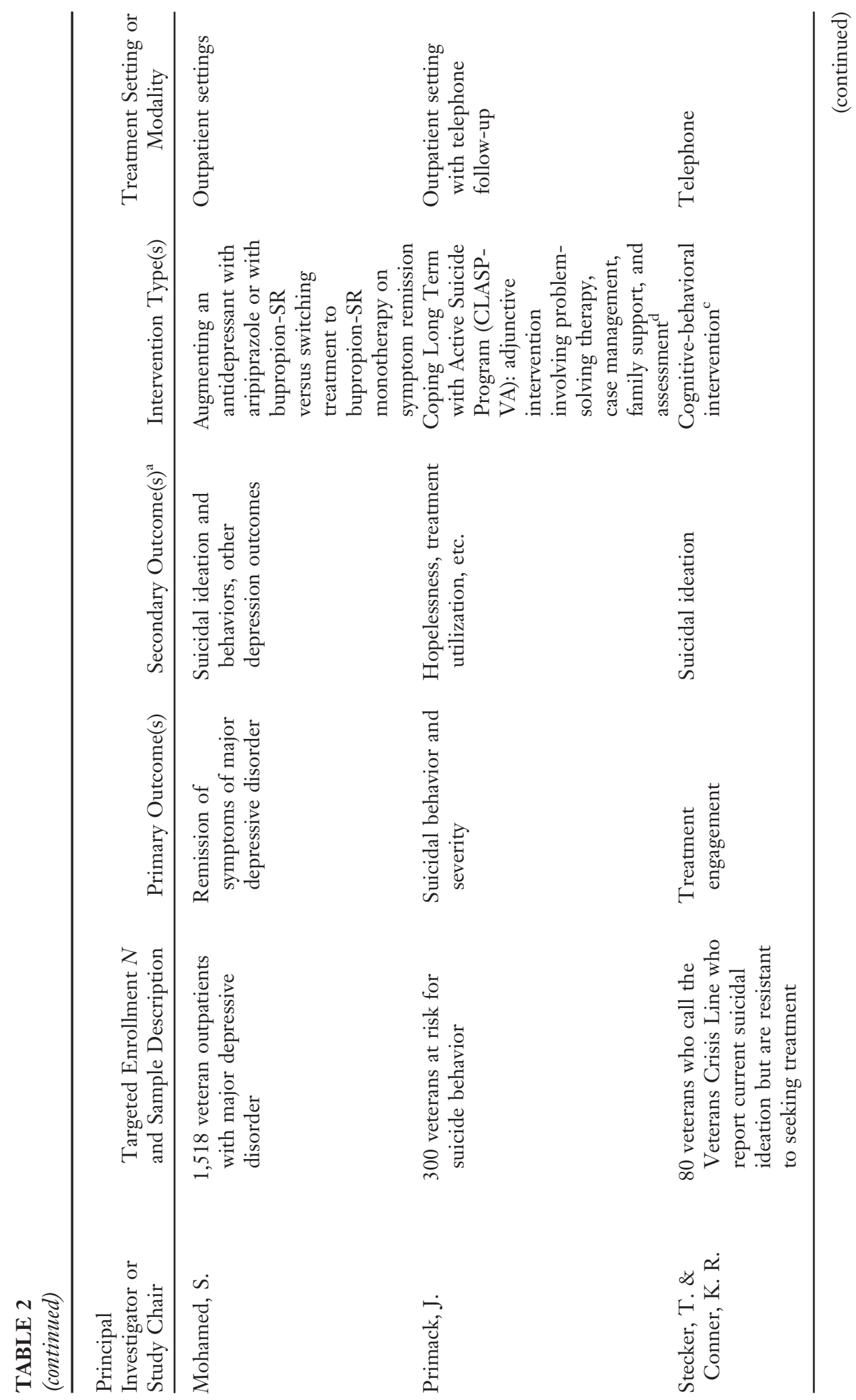




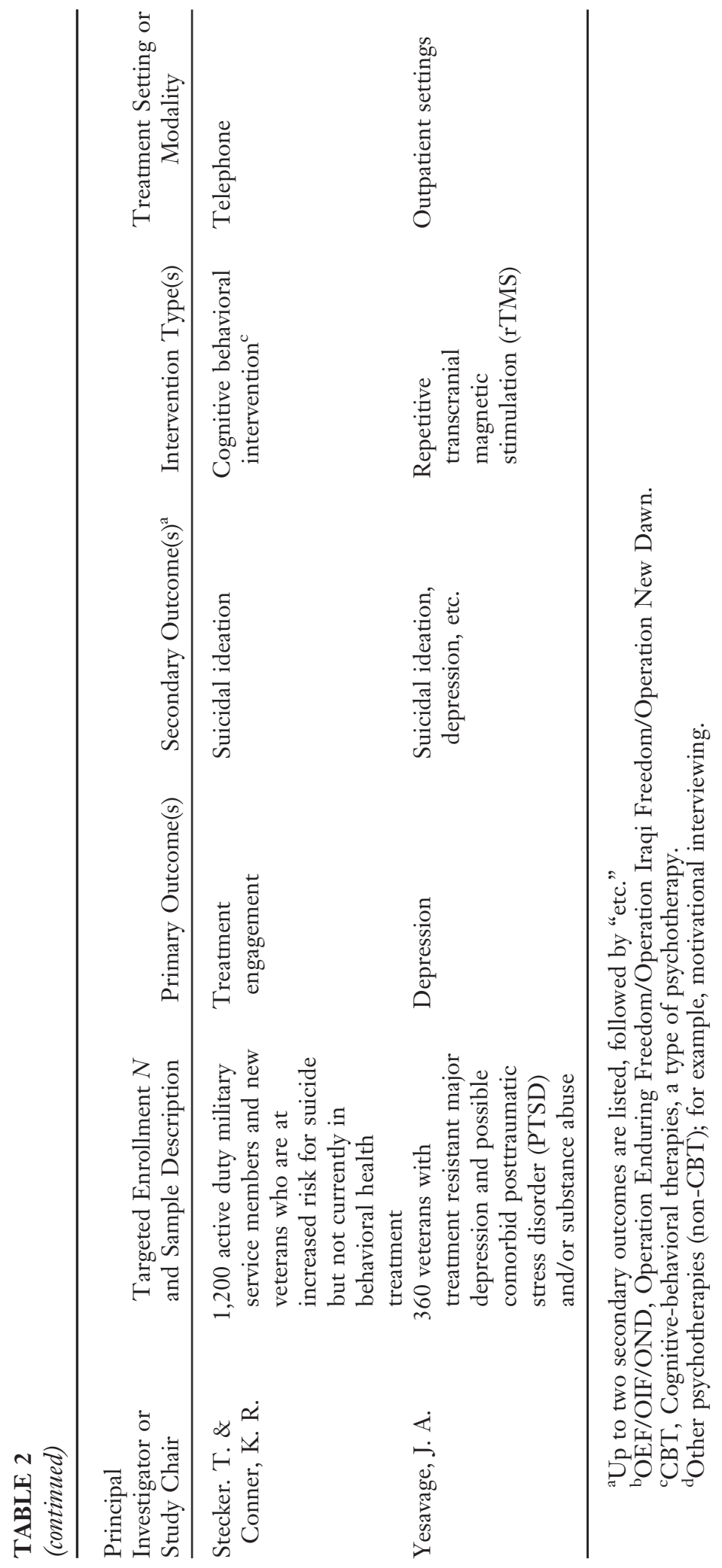


ting or modality. The primary source of data for the table was each identified study's page within ClinicalTrials.gov. Sources of innovation may be discerned from Table 2 through identification of departures or modernizations of typical practices concerning outcomes, interventions, and the modality or setting for treatment.

\section{Innovations in Outcomes}

Most of the investigations identified use suicidal ideation or a risk factor (e.g., depressive symptoms) as the primary outcome. The investigation "Caring Letters for Military Suicide Prevention" by Luxton (PI) (Luxton et al., 2014) stands out as the only study to use a primary outcome of suicide deaths. More specifically, the RCT uses a large $(N=4,730)$ and diverse high-risk clinical sample in order to compare caring letters delivered by e-mail (plus usual care) to usual care control condition. The letters are "simple expressions of care" delivered over defined intervals for 2 years following an inpatient hospitalization and include contact information for health services. Suicide attempts are not typically used as a primary outcome, an exception being the studies by Holloway (PI) of repeat suicide attempts (see Table 2). For example, in "Post Admission Cognitive Therapy (PACT) for the Inpatient Treatment of Military Personnel with Suicidal Behaviors," repeat suicide attempts are assessed over a 12-month follow-up period.

\section{Innovations in Interventions}

Several psychotherapy studies examine a cognitive-behavioral treatment or a closely related cognitive intervention; despite this, use of a widely used treatment approach does not rule out innovation. For example, "Mindfulness-based Cognitive Therapy for Preventing Suicide in Military Veterans" (Kline, PI) integrates mindfulness meditation techniques with cognitive therapy in a group format. There is also innovative psychotherapy work being done with motivational interviewing. For example, to our knowledge,
"Motivational Interviewing to Prevent Suicide in High Risk Veterans" (Britton, PI) is the first RCT to test a motivational interviewing intervention that was developed explicitly to address suicidal thoughts or behavior (Britton, Conner, \& Maisto, 2012) as opposed to other targets such as psychopathology risk factors.

Most of the investigations identified use a form of psychotherapy (including brief formats), although there are exceptions including studies of means of medication dispensing, pharmacotherapy, and nutritional supplement. While means restriction is an element of some of the treatments identified, "Blister Packaging Medication to Increase Treatment Adherence and Clinical Response (BP)" (Gutierrez, PI) is the only intervention with a singular focus on means restriction. "Lithium for Suicidal Behavior in Mood Disorders (Li+)" (Katz, PI) is one of only two pharmacotherapy studies identified. The project is also exceptional for its focus on time to suicide-related behavior. "The Better Resiliency among Veterans with Omega-3's (BRAVO) Study" (Marriott, PI) is the only study identified to use a nutritional intervention, a commercially available Omega-3 formulated juice (versus placebo juice) taken thrice daily over 6 months, in an examination of at-risk veteran outpatients.

Innovations in Intervention Settings and Treatment Modalities

Although most of the studies identified deliver treatments face-to-face in traditional clinical settings, there are exceptions. "Increasing Treatment Seeking Among At-Risk Service Members Returning from Warzones" (Stecker \& Conner, co-PIs) uses a one session cognitive-behavioral intervention that is rare in that it was developed and tested exclusively by phone. "The Military Continuity Project" (Comtois, PI) delivers caring text messages to provide supportive contact and practical information regarding behavioral health and crisis services. "Virtual Hope Box-Effectiveness of a Smartphone App for Coping with Suicidal Ideation" 
(Bush, PI) examines the efficacy of a smartphone app that contains elements of a hope box, a personalized strategy to remind individuals of their reasons for living, along with elements of evidence-based psychotherapies including dialectical behavior therapy.

\section{DISCUSSION}

Active suicide intervention research studies in military populations show innovations in the intervention types, settings, and modalities used and the primary outcomes studied. Opportunities for further innovations include, but are not limited to, increased focus on protective factors, means restriction, and "upstream" interventions. None of the studies identified examined a protective factor as the primary outcome despite calls for increased study of protective factors in suicide research (National Action Alliance for Suicide Prevention: Research Prioritization Task Force, 2014). Tempering this concern, in some investigations factors that may be hypothesized to be protective (e.g., reasons for living) are examined as a secondary outcome. Note that we limited the listing of secondary outcomes in Table 2 to two outcome measures and, therefore, some variables that may serve as protective factors under study are not listed.

Only one study that we identified focuses on means safety per se (Gutierrez, PI). The study is also noteworthy because the blister packaging of medications is done by providers prior to dispensing and, by doing so, there are likely to be fewer barriers to implementation than commonly observed with more complex and labor intensive therapy-based treatments (Kilbourne, Neumann, Pincus, Bauer, \& Stall, 2007). Interventions focused on firearm safety are also a critical direction for means safety research in military populations (Bush et al., 2013). Along these lines, there are well-conceived protocols for means safety counseling including addressing firearms (Bryan, Stone, \& Rudd, 2011) that require formal testing with military populations.
Although we identified examples of intervention studies delivered in nontraditional ways including by telephone, text message, and smartphone app, these were exceptions to the rule, with many interventions relying on face-to-face encounters between providers and patients in behavioral health treatment settings. Drawbacks to the latter paradigm include that most military service members and veterans who die by suicide are not in behavioral health treatment at the time of suicide (Britton, Ilgen, valenstein, Claassen, \& Conner, 2012; Bush et al., 2013). Military service members and veterans may resist agreeing to behavioral health treatment for a variety of reasons, including doubts about the helpfulness of treatment and one's ability to use it, anticipation of being offered medications but little else, concern about the impact of help-seeking on one's career, and practical barriers with transportation or scheduling (Stecker, Fortney, Hamilton, \& Ajzen, 2007). Therefore, it is critical that intervention research using nontraditional intervention venues and modalities be accelerated, including that which takes advantage of innovations in technology and communication.

Another innovative direction to take in future research is to increase the focus on outcomes that occur upstream from the development of suicidal thoughts and behavior, consistent with the idea that even minimal interventions delivered at an earlier stage may help channel individuals toward a path toward health and resilience and away from psychopathology and suicidal behavior (Caine, 2013). For example, research of the "good behavior game," a classroom team-based behavior management intervention, has shown that children in classrooms assigned to the intervention have lower risk for suicidal thoughts and attempts by young adulthood (Wilcox et al., 2008). Unfortunately, this type of early intervention study would not be uncovered by our search (i.e., RCTs rarely list outcomes that will be measured after several years). Overall, there are few data on the impact of upstream interventions on subsequent risk for suicidal behavior including in military families. 


\section{Limitations}

Innovation is merely one aspect of the quality of a study and that which is innovative may be flawed in other important respects (e.g., soundness of methodology). There is an inherent tension between the push for innovation and use of more established approaches that create the need for balancing risk and reward both in the design of studies and in funding decisions. Innovations may also be approached from other angles not considered in this review;

\section{REFERENCES}

Assessment and Management of Risk for Suicide Working Group. (2013). VA/DoD Clinical practice guideline for assessment and management of patients at risk for suicide. Retrieved April 14, 2014, from http://www.healthquality.va.gov/guidelines/ MH/srb/VADODCP_SuicideRisk_Full.pdf.

Britton, P. C., Conner, K. R., \& Maisto, S. A. (2012). An open trial of motivational interviewing to address suicidal ideation (MI-SI) with psychiatrically hospitalized veterans. Fournal of Clinical Psychology, 68, 961-967. doi:10.1002/ jclp. 21885

Britton, P. C., Ilgen, M. A., Valenstein, M., Claassen, C., \& Conner, K. R. (2012). Differences between veteran suicides with and without psychiatric symptoms. American Fournal of Public Health, 102(Suppl 1), S125S130.

Bryan, C. J., Stone, S. L., \& Rudd, M. D. (2011). A practical, evidence-based approach for means-restriction counseling with suicidal patients. Professional Psychology, 42, 339-346. doi: $10.1037 / \mathrm{a} 0025051$

Bush, N. E., Reger, M. A., Luxton, D. D., Skopp, N. A., Kinn, J., Smolenski, D., ET AL. (2013). Suicides and suicide attempts in the U.S. military, 2008-2010. Suicide and Life-Threatening Behavior, 43, 262-273. doi:10.1111/sltb.12012

CaIne, E. D. (2013). Forging an agenda for suicide prevention in the United States. American Fournal of Public Health, 103, 822-829. doi:10.2105/AJPH.2012.301078

Innovation. (2014). In Merriam-Webster. com. Retrieved February 7, 2014, from http:// www.merriam-webster.com/dictionary/innovation

Kilbourne, A. M., Neumann, M. S., Pincus, H. A., Bauer, M. S., \& Stall, R. for example, through advances in the testing of theorized mechanisms of change (Nock, 2007). We provided a sampling of innovative practices within our definition, not an exhaustive review. Accordingly, the fact that we did not discuss a study should not be interpreted to indicate that the study lacked innovation. As with any search, there is the potential for missed studies. Studies that did not meet our eligibility criteria, including internally funded pilot projects, demonstration projects, and completed RCTs, may feature noteworthy innovations.
(2007). Implementing evidence-based interventions in health care: Application of the replicating effective programs framework. Implementation Science, 2, 42. doi:10.1186/1748-5908-2-42

Luxton, D. D., Thomas, E. K., Chipps, J., Relova, R. M., Brown, D., McLay, R., ET AL. (2014). Caring letters for suicide prevention: Implementation of a multi-site randomized clinical trial in the U.S. military and Veteran Affairs healthcare systems. Contemporary Clinical Trials, 37, 252-260.

National Action Alliance for Suicide Prevention: Research Prioritization Task Force. (2014). A prioritized research agenda for suicide prevention: An action plan to save lives. Rockville, MD: National Institute of Mental Health and the Research Prioritization Task Force.

Nock, M. K. (2007). Conceptual and design essentials for evaluating mechanisms of change. Alcoholism, 31(Supp 3), 4-12. doi:10.1111/ j.1530-0277.2007.00488.x

Stecker, T., Fortney, J. C., Hamilton, F., \& AJZEN, I. (2007). An assessment of beliefs about mental health care among veterans who served in Iraq. Psychiatric Services, 58, 1358-1361. Wilcox, H. C., Kellam, S. G., Brown, C. H., Poduska, J., Ialongo, N. S., Wang, W., ET AL. (2008). The impact of two universal randomized first- and second-grade classroom interventions on young adult suicide ideation and attempt. Drug Alcohol Dependence, 95(Suppl 1), S60-S73.

Manuscript Received: April 22, 2014

Revision Accepted: July 21, 2014 